\title{
Correction: Zbtb16 regulates social cognitive behaviors and neocortical development
}

Noriyoshi Usui (D, Stefano Berto, Ami Konishi, Makoto Kondo, Genevieve Konopka, Hideo Matsuzaki id and Shoichi Shimada

\section{Correction to: Translational Psychiatry https://doi.org/10.1038/s41398-021-01358-y published online 24 April 2021}

The original version of this article unfortunately contained a mistake in the following sentence "These analyses indicate that the Ztbt16-regulated transcriptome is related to both ASD and SCZ". "Ztbt16" should be changed to "Zbtb16". We apologize for the error. The original article has been corrected.

Published online: 11 May 2021 